\title{
Studies of ${ }^{139} \mathrm{La}$ and ${ }^{63 / 65} \mathrm{Cu}-\mathrm{NQR}$ in $\mathrm{La}_{2-x} \mathrm{M}_{x} \mathrm{CuO}_{4}$ $(\mathbf{M}=\mathbf{B a}, \mathbf{S r})$ *
}

\author{
K. Kumagai, Y. Nakamura, I. Watanabe, and H. Nakajima \\ Department of Physics, Faculty of Science, Hokkaido University, Sapporo 060, Japan
}

Z. Naturforsch. 45a, 433-438 (1990); received September 19, 1989

\begin{abstract}
The nuclear quadrupole resonance of ${ }^{139} \mathrm{La}$ and ${ }^{63 / 65} \mathrm{Cu}$ in $\mathrm{La}_{2-x} \mathrm{M}_{x} \mathrm{CuO}_{4}(\mathrm{M}=\mathrm{Ba}, \mathrm{Sr})$ is investigated for $x$ up to unity. The shift of the resonance frequency of ${ }^{x}{ }^{139}$ La-NQR for $0.01<x<0.05$ indicates an anomalous change of the quadrupole interactions at $T_{c}^{*}$, where the frustrated magnetic phase appears. The Cu-NQR reveals clear changes of $T_{1}^{-1}$ and $T_{2}^{-1}$ and also of the profile of the $\mathrm{Cu}-\mathrm{NQR}$ spectra with increasing $x$ beyond $x=0.3 \sim 0.4$, suggesting a suppression of antiferromagnetic fluctuations by hole-doping, and also a change of the electronic state of the La-system around $x=0.3 \sim 0.4$.
\end{abstract}

Key words: NQR High- $T_{\mathrm{c}}$ superconductivity, $\mathrm{La}_{2-x} \mathrm{M}_{x} \mathrm{CuO}_{4}$.

\section{Introduction}

After the discovery of copper based oxide superconductors [1], many families differing in structure and constituent elements have been found. Strikingly they all share $\mathrm{Cu}-\mathrm{O}$ planes as two dimensional substructures and commonly antiferromagnetism near the superconducting phase [2]. It is therefore very important to understand the strong antiferromagnetic spin fluctuations of the $\left(\mathrm{CuO}_{2}\right)$ sheets. Among various kinds of fundamental measurements, NMR techniques play a central role to elucidate the local electronic and magnetic properties of high $T_{\mathrm{c}}$-oxides [3].

Though $\mathrm{La}_{2-x} \mathrm{M}_{x} \mathrm{CuO}_{4} \quad\left(\mathrm{M}=\mathrm{Sr}^{2+}, \mathrm{Ba}^{2+}\right.$ and $\mathrm{Ca}^{2+}$ ) has a simple structure, this system involves rich physical properties such as magnetic, structural and metal-insulator transitions as a fuction of hole-doping [4]. In order to clarify the effect of doped holes on the characteristic spin and charge fluctuations under the strong electron correlations in the $\left(\mathrm{CuO}_{2}\right)$ sheets, intensive investigations of $\mathrm{Cu}$ - and La-NQR have already been performed [5-11]. In this paper we report on details of the temperature and $\mathrm{x}$-dependences of the ${ }^{63 / 65} \mathrm{Cu}-\mathrm{NQR}$ of $\mathrm{La}_{2-x} \mathrm{Sr}_{x} \mathrm{CuO}_{4}$ up to $x=1$. Extended results of the ${ }^{139} \mathrm{La}-\mathrm{NQR}$ in a small region of Ba-doping are also briefly reported.

* Presented at the Xth International Symposium on Nuclear Quadrupole Resonance Spectroscopy, Takayama, Japan, August 22-26, 1989.

Reprint requests to Dr. K. Kumagai, Department of Physics, Faculty of Science, Hokkaido University, Sapporo 060, Japan

\section{Experimental}

All samples were prepared by solid state reaction from mixed powders of $\mathrm{La}_{2} \mathrm{O}_{3}, \mathrm{BaCO}_{3}, \mathrm{SrCO}_{3}$, and $\mathrm{CuO}$ with $99.99 \%$ purity. For details of the preparation see [6]. The reacted pellets were crushed into 350-mesh powder for the NMR measurements. In agreement with the previous result that the $\mathrm{K}_{2} \mathrm{NiF}_{4}$ type compound $\mathrm{La}_{2-x} \mathrm{Sr}_{x} \mathrm{CuO}_{4}$ can be synthesized with $x$ up to 1.34 [12], we confirmed by $\mathrm{x}$-ray diffraction measurements that the present samples are crystallized in a single phase. The lattice constant of the $c$-axis of the present samples increases with increasing $x$ up to $x \sim 0.3$ and then decreased rapidly for $x>0.4[12,13]$. The superconducting transition temperatures were obtained by resistivity and magnetic susceptibility measurements [14]. Bulk superconductivity was also confirmed in the range of $0.08 \leqq x$ $\leqq 0.25$ by the heat capacity jump at $T_{\mathrm{c}}$ [15]. A conventional phase coherent pulse NMR apparatus was used for observing the relaxation and NQR spectra of the ${ }^{139} \mathrm{La}$ and ${ }^{63 / 65} \mathrm{Cu}$ nuclei. The nuclear spin-lattice relaxation time, $T_{1}$ was measured by the saturating comb pulse method.

\section{Results and Discussion}

Undoped $\mathrm{La}_{2} \mathrm{CuO}_{4}$ is an insulating antiferromagnet of $T_{\mathrm{N}} \sim 270 \mathrm{~K}$. The system remains insulating for small hole-doping ( $\mathrm{Sr}$ concentration) up to $x=0.05$. On passing $x=0.05$, superconductivity appears in the range $0.05<x<0.25$ [16], where the normal state re-

0932-0784 / $90 / 0300-0433 \$ 01.30 / 0$. - Please order a reprint rather than making your own copy. 


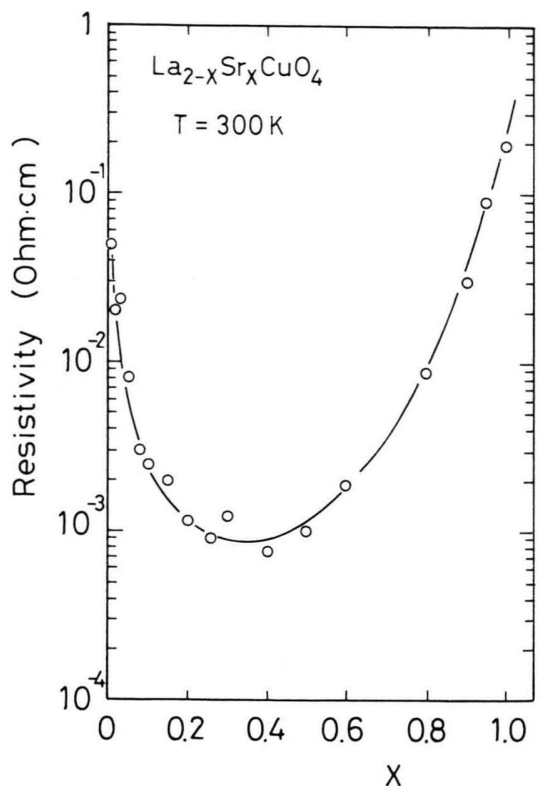

Fig. 1. X-dependence of the electrical resistivity of $\mathrm{La}_{2-x} \mathrm{Sr}_{x} \mathrm{CuO}_{4}$ at $300 \mathrm{~K}$.

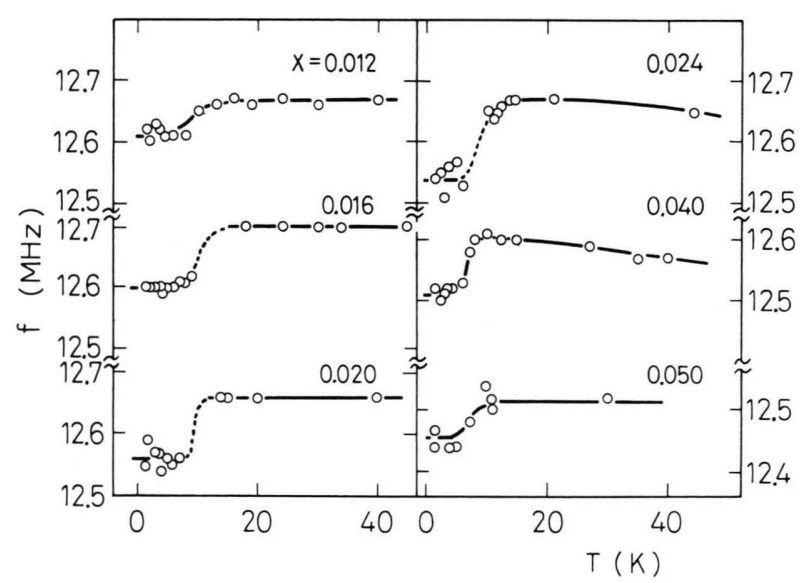

Fig. 2. Temperature dependence of the resonance frequency of ${ }^{139} \mathrm{La}$ of the $\pm 3 / 2 \leftrightarrow \pm 5 / 2$ transition in $\mathrm{La}_{2-x} \mathrm{Ba}_{x} \mathrm{CuO}_{4}$.

sistivity above $T_{\mathrm{c}}$ increases linearly with temperature, showing that the system is metallic. The resistivity at $300 \mathrm{~K}$, shown in Fig. 1, has a minimum near $x=$ $0.3 \sim 0.4$ and thereafter significantly with increasing $x$ up to $x=1$. The overall $\mathrm{x}$-dependence of the resistivity is anti-correlated with the $\mathrm{x}$-dependence of the lattice parameter [13].

As reported in the previous paper [6], the divergence of $T_{1}^{-1}$ of the ${ }^{139} \mathrm{La}-\mathrm{NQR}$ indicates the existence of a peculiar frustrated magnetic phase between the $3 \mathrm{D}$-antiferromagnetic $(x<0.02)$ and the superconducting $(x>0.06)$ phase at $T_{\mathrm{c}}^{*} \sim 10 \mathrm{~K}[6]$. This transition is accompanied with magnetic ordering [7]. The resonance frequency for the nuclear $\pm 3 / 2 \leftrightarrow \pm 5 / 2$ transition of ${ }^{139} \mathrm{La}$ is shown as a function of temperature in Figure 2. An anomalous shift of the resonance frequency of the ${ }^{139} \mathrm{La}-\mathrm{NQR}$ to lower frequencies is observed at the magnetic transition [6]. The difference of the resonance frequencies between above and below $T_{\mathrm{c}}^{*}$ is largest near $x=0.02$, where the enhancement of $T_{1}^{-1}$ is most pronounced [6]. Such anomalies are not observed at the 3D-dimensional antiferromagnetic transition temperatures for $x<0.02$. From a detailed investigation of the resonance frequencies corresponding to the other $\pm 1 / 2 \leftrightarrow \pm 3 / 2$ and $\pm 5 / 2 \leftrightarrow \pm 7 / 2$ transitions we obtain the parameters of the electric quadrupole interactions and the internal magnetic field by a numerical solution of the Hamiltonian $H=H_{\mathrm{Q}}+H_{\mathrm{hf}}$ with

$$
\begin{array}{r}
H_{\mathrm{Q}}=\frac{e^{2} q Q}{4 I(2 I-1)}\left[3 I_{z}^{2}-I(I+1)+\eta / 2\left(I_{+}^{2}+I_{-}^{2}\right)\right], \\
H_{\mathrm{hf}}=-\gamma h H_{0}\left[I_{z} \cos \theta+1 / 2 \sin \theta\left(I_{+} \exp (-i \phi)\right.\right. \\
\left.\left.+I_{-} \exp (i \phi)\right)\right],
\end{array}
$$

where $v_{\mathrm{q}}$ is the quadrupole frequency, $\eta$ the asymmetry parameter of the electric field gradient (EFG), and $\theta$ and $\phi$ are the angles between the principal axes of the EFG and the internal magnetic field, $H_{0}$.

Figure 3 shows the $\mathrm{x}$-dependences of $v_{\mathrm{Q}}$ and $\eta$ above and far below $T_{\mathrm{c}}^{*}$ in $\mathrm{La}_{2-x} \mathrm{Ba}_{x} \mathrm{CuO}_{4} \cdot v_{\mathrm{Q}}$ decreases gradually with $x$ and changes little at $T_{\mathrm{c}}^{*}$. The primary cause of the frequency shift with temperature is attributed to the change of $\eta$. The values of $\eta$ in the peculiar magnetic phase are smaller than the ones in the paramagnetic phase above $T_{\mathrm{c}}^{*}$. This fact implies that the magnetic order of $\mathrm{Cu}$ moments below $T_{\mathrm{c}}^{*}$ is associated with an anomalous change of electric quadrupole interactions. The main source of the EFG is considered to be intra-atomic in origin, i.e. an aspherical distribution of the valence electrons [17]. The change of the electronic state owing to the charge transfer may modify the electronic configuration at La sites in the peculiar frustrated magnetic phase. A magnetic origin such as magnetostriction below $T_{\mathrm{c}}^{*}$ can be considered. The possible origin of the anomalies will be discussed separately by means of the detailed analysis of ${ }^{139} \mathrm{La}$ NQR [18].

Spin echo signal of ${ }^{63 / 65} \mathrm{Cu}$ are observed above $30 \mathrm{MHz}$ in zero external field for $x \geqq 0.12[9,10]$. The 


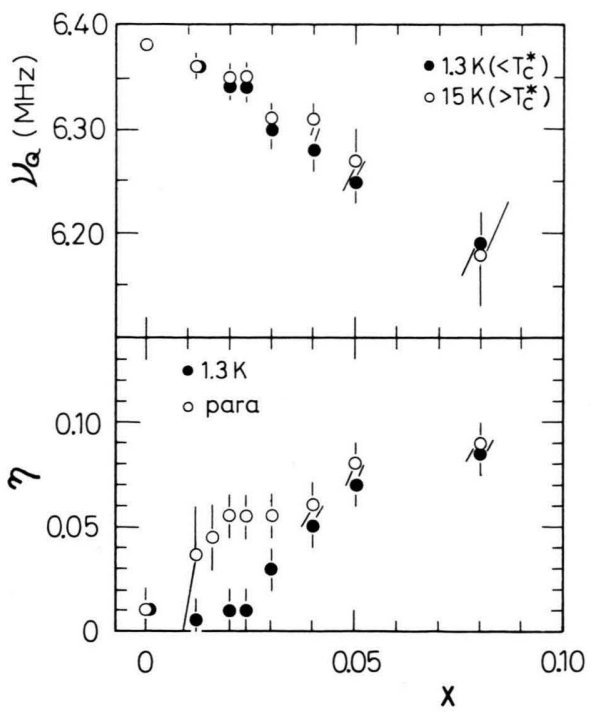

Fig. 3. X-dependences of the resonance frequency, $v_{\mathrm{Q}}$, and the asymmetry parameter, $\eta$, of the electric field gradient in the magnetic phase (closed symbols) and the paramagnetic phase (open symbols) of $\mathrm{La}_{2-x} \mathrm{Ba}_{x} \mathrm{CuO}_{4}$.

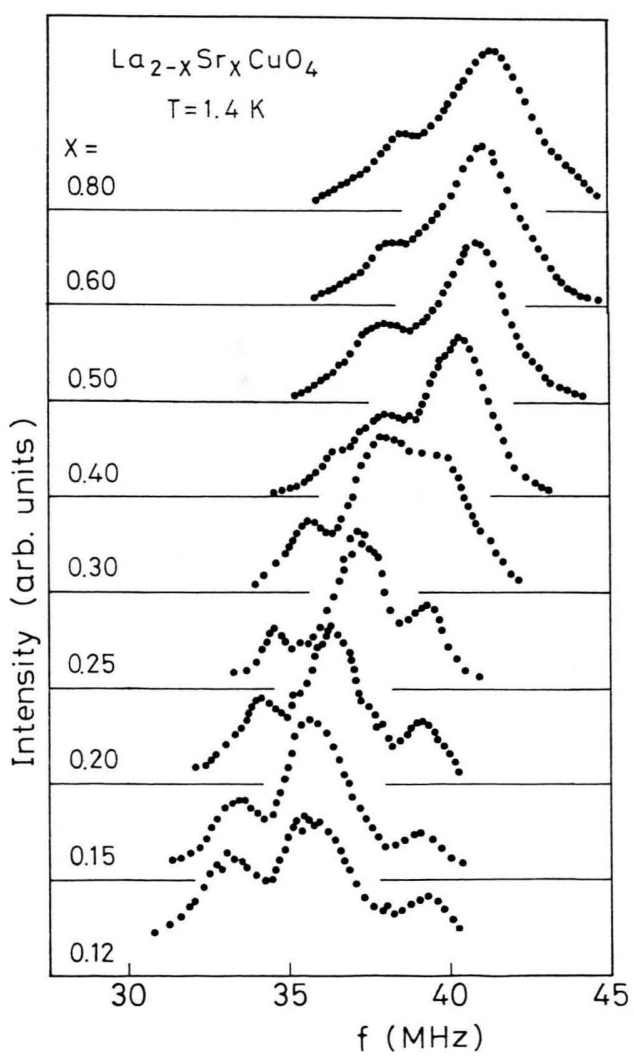

Fig. 4. Spin echo spectra of ${ }^{63 / 65} \mathrm{Cu}$ in $\mathrm{La}_{2-x} \mathrm{Sr}_{x} \mathrm{CuO}_{4}$ at $1.4 \mathrm{~K}$. failure of the observation of the NQR signals for the interesting region $x \leqq 0.1$ is caused by their very shortened spin-spin relaxation time. Figure 4 shows the zero field spectra of ${ }^{63 / 65} \mathrm{Cu}$ at $1.4 \mathrm{~K}$ for various $x$ values. As reported previously [10,13], the $\mathrm{Cu}$-spectra for $x \leqq 0.3$ consist of three peaks, while the spectra for $x \geqq 0.4$ show only two peaks corresponding to the two $\mathrm{Cu}$ isotopes. The line profiles are not modified by variations in the sample preparation. The spectra are confirmed by the studies $[19,20]$. Magnetic or electrical quadrupole one origins have been discussed. Recently the complex spectra were attributed to the distribution of the electric quadrupole interactions due to the oxygen vacancies and/or the random distribution of $\mathrm{Sr}$ and $\mathrm{La}$ around the $\mathrm{Cu}$ ions $[19,20]$. The effect of the charge differentiation on the EFG is proposed to be similar to that in the $Y$ system [20].

The nuclear spin-lattice relaxation rate $T_{1}^{-1}$ was measured for the largest peak in each spectrum. The recovery curve of the nuclear magnetization after saturation is almost single exponential around $x=0.4$ from 1.5 to $100 \mathrm{~K}$. However, non-exponential behavior appears for $x \leqq 0.3$ and $x \geqq 0.5$. The non-exponential behavior in the superconducting samples becomes more significant with decreasing temperature. The profile of the recovery curve does not depend on the saturating condition. Therefore, the distribution in $T_{1}$ is not due to the spin diffusion process. As each component of the magnetization recovery curve changes similarly with temperature, the temperature dependence of $T_{1}$ is considered to change similarly for each component. Thus we show, for simplicity, the temperature dependence of the longest component of $T_{1}^{-1}$, which is extracted from the tail part of the recovery curves.

As shown in Fig. 5, the temperature dependence of $T_{1}^{-1}$ in the superconducting sample with $x \leqq 0.25$ is very similar to that of $\mathrm{Cu}$ in the $\left(\mathrm{CuO}_{2}\right)$ plane of the $Y$-system [21], namely $T_{1}^{-1}$ shows a sharp decrease without the BCS enhancement just below $T_{\mathrm{c}}$, and decreases power-low-like with decreasing temperature. Quite similar results in the La-system are confirmed by independent investigations $[7,19,20]$. This behavior is very anomalous in comparison with the BCS type superconductors.

With increasing temperature above $T_{\mathrm{c}}, T_{1}^{-1}$ is much enhanced and reaches almost the order of $\mathrm{ms}^{-1}$ in the normal state for $x=0.2$. For $x \geqq 0.3$, where the electrical resistivity shows metallic behavior, the temperature dependence of $T_{1}^{-1}$ obeys the Korringa rela- 


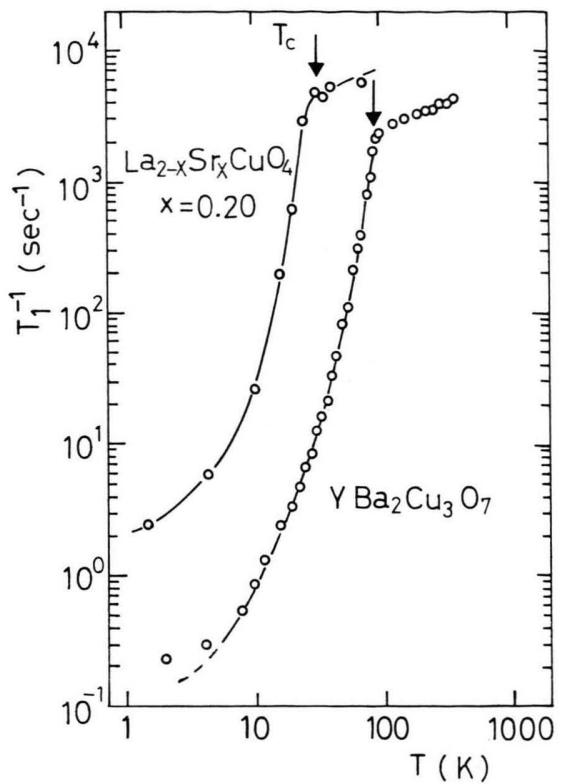

Fig. 5. Temperature dependence of the nuclear spin-lattice relaxation rate, $T_{1}^{-1}$, of ${ }^{63} \mathrm{Cu}$ in the superconducting $\mathrm{La}_{1.8} \mathrm{Sr}_{0.2} \mathrm{CuO}_{4}$ and in $\mathrm{YBa}_{2} \mathrm{Cu}_{3} \mathrm{O}_{7}$ (from [21]).

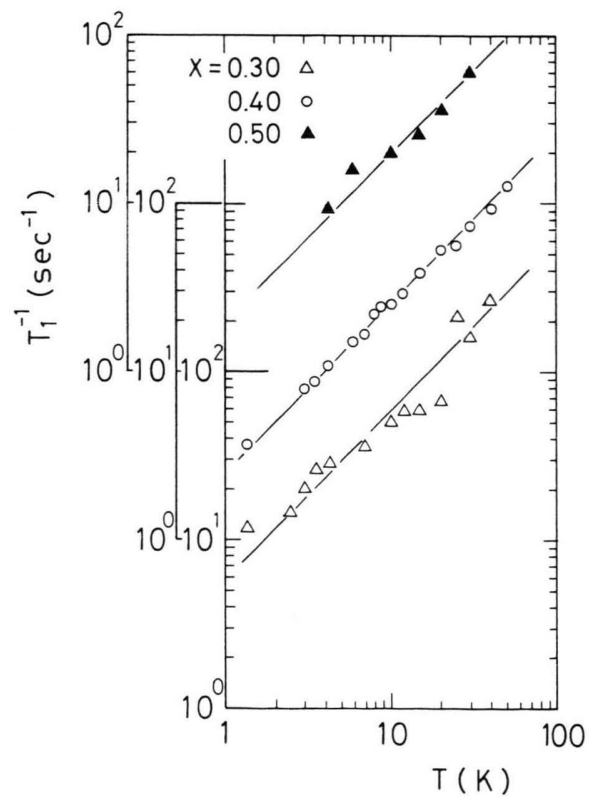

Fig. 6. Temperature dependence of $T_{1}^{-1}$ of ${ }^{63} \mathrm{Cu}$ in the normal metallic samples of $\mathrm{La}_{2-x} \mathrm{Sr}_{x} \mathrm{CuO}_{4}$.

tion $T_{1} T=$ const between 1.5 and $100 \mathrm{~K}$, as shown in Figure 6 . (For $x \geqq 0.6$, the deviation from the Korringa relation becomes substantial below $30 \mathrm{~K}$. In this case, we deduce simply the $T_{1} T$ values from the narrow range of high temperatures.) This indicates that the

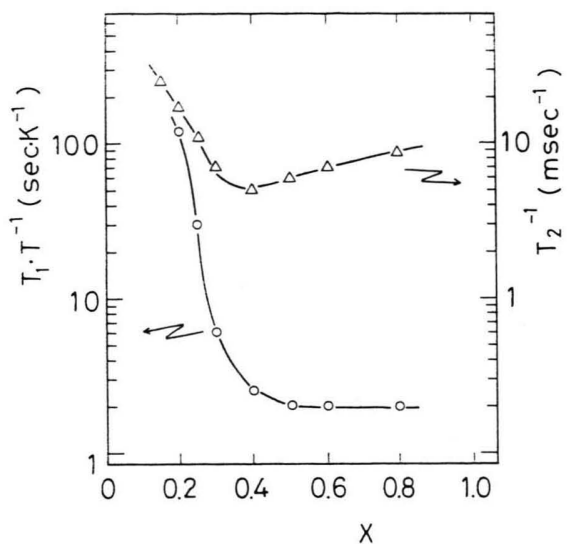

Fig. 7. X-dependence of $\left(T_{1} T\right)^{-1}(0)$ and the spin-spin relaxation rate $T_{2}^{-1}(\Delta)$ of ${ }^{63} \mathrm{Cu}$ in $\mathrm{La}_{2-x} \mathrm{Sr}_{x} \mathrm{CuO}_{4}$ at $77 \mathrm{~K}$.

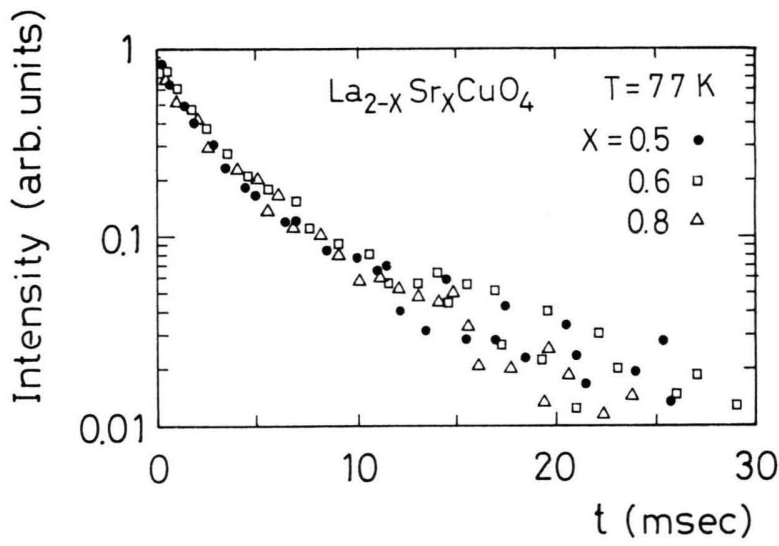

Fig. 8. Nuclear magnetization recovery data as a function of pulse separation time in $\mathrm{La}_{2-x} \mathrm{Sr}_{x} \mathrm{CuO}_{4}$ of $x=0.5,0.6$, and 0.8 .

electronic state is metallic and that the thermal excitation of conduction electrons at the Fermi level is sizable at the $\mathrm{Cu}$ sites. Figure 7 shows the $x$-dependence of $\left(T_{1} T\right)^{-1}$ in the normal state. $\left(T_{1} T\right)^{-1}$ decreases dramatically with $x$ up to $x=0.3$ and attains the constant value $\sim 2.0(\mathrm{~s} \mathrm{~K})^{-1}$ for $x \geqq 0.4$. To see the independence of $T_{1}$ of $x$ for $x \geqq 0.4$, we show the recovery curves at $77 \mathrm{~K}$ for $x=0.5,0.6$, and 0.8 in Figure 8. The data points scatter around the same curve within the entire range of time, showing that both the short and long components of $T_{1}$ are essentially independent of $x$ for $x \geqq 0.4$.

In addition to the remarkable change of $T_{1}$, the spin-spin relaxation rate, $T_{2}^{-1}$, decreases rapidly with increasing $x$ up to $x=0.3$, as shown in Figure 7. For $x \geqq 0.4, T_{2}^{-1}$ increases slightly with $x . T_{2}$ is usually determined by dipole coupling between $\mathrm{Cu}$ nuclear 
spins and is expected to remain nearly constant in the order of ms because of the unchanged nuclear number and distance between $\mathrm{Cu}$ nuclei on Sr-doping. Therefore, the enhanced $T_{2}^{-1}$ is to be attributed to another origin. In $[10,13]$, we suggested that the shortened $T_{2}$ originates from the indirect nuclear coupling via virtual antiferromagnetic spin excitations. Recently Pennington et al. [22] showed that $T_{2}$ of $\mathrm{Cu}$ (II) in $\mathrm{YBa}_{2} \mathrm{Cu}_{3} \mathrm{O}_{7}$ can be explained by a strong indirect coupling between $\mathrm{Cu}$ (II) nuclear spins through the nuclear moments which are coupled through superexchange interactions to each other. In the La-system, $T_{2}$ increases from $20 \mu \mathrm{s}$ for $x=0.12$ to $200 \mu \mathrm{s}$ for $x=0.3$, as shown in Figure 7. The large enhancement of $T_{2}$ and $T_{1}$ is considered to evidence the $\mathrm{Cu}$ nuclear spin-spin coupling through $\mathrm{Cu}$ electronic moments.

There rapid decreases of $T_{1}^{-1}$ and $T_{2}^{-1}$ with increasing $x$ suggest the suppression of the antiferromagnetic fluctuations by hole-doping, and that the suppressed relaxation for $x>0.4$ can be considered to result from the diminuition of the effective magnetic moments by hole-doping. Possibly the doped hole spins on the oxygen ions form nonmagnetic singlet states with adjacent $\mathrm{Cu}^{2+}$ spins which have no magnetic interaction with other d-holes [23]. In this case, the anomalous splitting of the NQR spectra is not expected any more. It should be emphasized that the clear difference of the $x$-dependencies of $T_{1}$ and $T_{2}$ must be related with the change of the electronic state beyond $x=0.3 \sim 0.4$ in the La-system. The quantities such as susceptibility, Hall coefficient and thermoelectric power change significantly near $x=0.3$ [24], implying that the electronic states in the normal state are much altered by hole doping near $x=0.3$. The decrease of $T_{\mathrm{c}}$ for $x>0.2$ seems to occur concurrently with the change of the normal state quantities. Thus, one may safely say that the $\mathrm{Cu}-\mathrm{NQR}$ of the La-system provides another evidence of the anomalous change of the magnetic state of $\mathrm{Cu}$ spins by hole-doping around $x=0.3 \sim 0.4$.

For the more concentrated region of $x \geqq 0.8$, the electrical resistivity shows semiconducting behavior [13]. The temperature dependence of the resistivity for $x>0.8$ is apparently fitted by the relation $\log \varrho \propto$ $\exp \left(T^{-1 / 4}\right)$ below $300 \mathrm{~K}$ [13], suggesting that the variable-range hopping conduction is dominant near $x=1$, and that the electronic states are localized near the Fermi energy. In this concentrated region of $x \geqq 0.8$, the anomalies in the temperature dependence of the $T_{1}^{-1}$ appear at low temperatures, as shown in Figure 9. The temperature at which $T_{1}^{-1}$ shows a peak

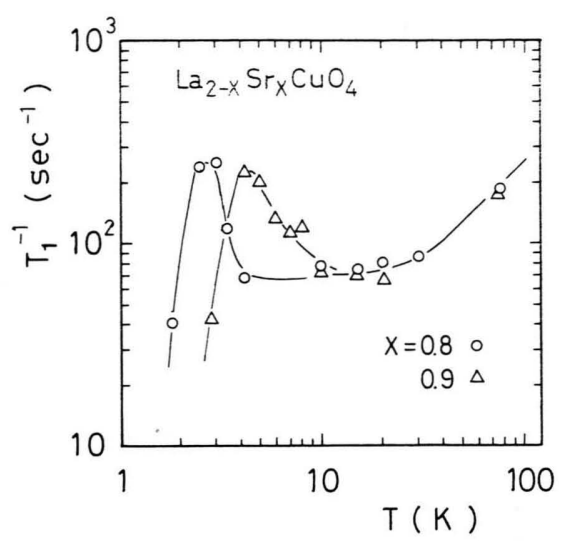

Fig. 9. Temperature dependence of $T_{1}^{-1}$, of ${ }^{63} \mathrm{Cu}$ in the semiconducting samples of $\mathrm{La}_{2-x} \mathrm{Sr}_{x} \mathrm{CuO}_{4}$ with $x=0.8$ and 0.9 .

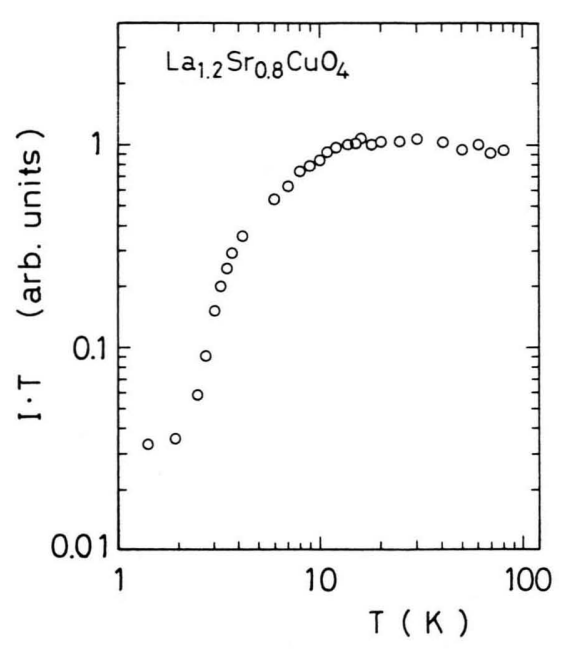

Fig. 10. Intensity of the Cu-NQR times temperature, $I \cdot T$, for $\mathrm{La}_{1.2} \mathrm{Sr}_{0.8} \mathrm{CuO}_{4}$ as a function of temperature.

increases with increasing $x$. In addition to the relaxation anomalies, the intensity of the spectra decreases significantly near the peak temperature in nuclear relaxation, as shown in Figure 10. Moreover, the intensities for the samples of $x=0.95$ and 1 are almost wiped out from the range of around $35-50 \mathrm{MHz}$ at least below $77 \mathrm{~K}$. The enhancement of $T_{1}^{-1}$ and the loss of the NQR signals suggest that a kind of magnetic order (possibly antiferromagnetic) again appears for $x \geqq 0.8$, similar as in the undoped and dilutelydoped region, where the system is insulating (semiconducting). The magnetic ordering temperatures are 2.5 and $4 \mathrm{~K}$ for $x=0.8$ and $x=0.9$, respectively, and are expected to be far above $77 \mathrm{~K}$ for $x \geqq 0.95$. 


\section{Summary}

We have investigated ${ }^{139} \mathrm{La}$ and ${ }^{63 / 65} \mathrm{Cu}-\mathrm{NQR}$ in a wide range of $\mathrm{Sr}$-substitution in $\mathrm{La}_{2-x} \mathrm{Sr}_{x} \mathrm{CuO}_{4}$. The divergence of the nuclear relaxation rates of ${ }^{139} \mathrm{La}$ indicates that a frustrated magnetic phase exists between the antiferromagnetic and the superconducting region. The anomalous change of the electric quadrupole interaction at the La sites is accompanied with the generation of magnetic internal fields. The asymmetry, $\eta$, of the electric quadrupole interaction becomes negligibly small below $T_{\mathrm{c}}^{*}$, implying that the electronic configurations change with the peculiar magnetic transition.

Splitted spectra of ${ }^{63 / 63} \mathrm{Cu}-\mathrm{NQR}$ are observed for $0.12 \leqq x \leqq 0.3$, while the spectra show only one peak

[1] J. D. Bednorz and K. A. Muller, Z. Phys. B 64, 189 (1986).

[2] See e.g. Y. Kitaoka et al., J. Phys. Soc. Japan 57374 (1988).

[3] See e.g. Proc. of Inter. Conf. of High- $T_{\mathrm{c}}$ Superconductors (1988, Interlaken), Physica C 154-155 (1988).

[4] See e.g. T. Fujita, Y. Aoki, Y. Maeno, J. Sakurai, H. Fukaba, and H. Fujii, Japan J. Appl. Phys. 26, L 368 (1987).

[5] Y. Kitaoka, H. Hiramatsu, T. Kohara, K. Asayama, K. Ohisahi, M. Kikuchi, and N. Kobayashi, Japan J. Appl. Phys. 26, L 397 (1987).

[6] I. Watanabe, K. Kumagai, Y. Naka, T. Kimura, Y. Nakamichi, and H. Nakajima, J. Phys. Soc. Japan 56, 3028 (1988), and K. Kumagai, I. Watanabe, H. Aoki, Y. Nakamura, T. Kimura, Y. Nakamichi, and H. Nakajima, Physica B 148, 480 (1987).

[7] Y. Kitaoka, K. Ishida, H. Hirmatsu, and K. Asayama, J. Phys. Soc. Japan 57, 734 (1938).

[8] H. Nishihara, H. Yasuoka, T. Shimizu, T. Tsuda, T. Imai, S. Sasaki, S. Kanbe, K. Kishio, K. Kitazawa, and K. Fueki, J. Phys. Japan 57, 4559 (1987).

[9] K. Ishida, Y. Kitaoka, and K. Asayama, J. Phys. Soc. Japan 59, 36 (1989).

[10] K. Kumagai and Y. Nakamura, Physica C 157, 307 (1989).

[11] T. Kobayashi, S. Wada, Y. Kitaoka, and K. Asayama, J. Phys. Soc. Japan 58, 2658 (1989). corresponding to each $\mathrm{Cu}$ isotope for $x \geqq 0.4$. In the superconducting samples of $x \leqq 0.25, T_{1}^{-1}$ shows a sharp decrease without the BCS type enhancement just below $T_{\mathrm{c}}$, and also a power-low-like decrease with decreasing temperature. In the normal state, the Korringa relation, $T_{1} T=$ const, is observed below $100 \mathrm{~K}$ for $x \geqq 0.4$.

\section{Acknowledgements}

This work was supported in part by a Grant-in-Aid for Scientific Research on Priority Areas "Mechanism of Superconductivity" from the Ministry of Education, Science and Culture of Japan.

[12] N. Nguyen, J. Choisnet, M. Hervieu, and B. Raveau, J. Solid State Chem. 39, 120 (1981).

[13] Y. Nakamura and K. Kumagai, Physica C 161, 265 (1989).

[14] K. Kumagai, Y. Nakamura, I. Watanabe, Y. Nakamichi, and H. Nakajima, J. Mag. Materials, 76-77, 601 (1988).

[15] N. Wada, H. Muro-oka, Y. Nakamura, and K. Kumagai, Physica C 157, 453 (1989).

[16] J. B. Torrance, Y. Tokura, A. I. Nazzal, A. Bezinge, T. C. Hung, and S. S. P. Parkin, Phys. Rev. Lett. 61, 1127 (1988).

[17] C. Amdrosch-Draxl, P. Blaha, and K. Schwarz, to be published in Physica C (1989).

[18] I. Watanabe and K. Kumagai, in preparation.

[19] K. Ishida, T. Kondo, Y. Kitaoka, and K. Asayama, J. Phys. Soc. Japan 58, 2638 (1989).

[20] K. Yoshimura, T. Imai, T. Simizu, Y. Ueda, K. Kosuge, and H. Yasuoka, to be published in J. Phys. Soc. Japan 58 (1989).

[21] T. Imai, T. Shimizu, Y. Yasuoka, Y. Ueda, and K. Kosuge, J. Phys. Soc. Japan 57, 2280 (1988).

[22] C. H. Pennington, D. J. Durand, C. P. Slichter J. P. Rice, E. D. Bukowski, and D. M. Ginsberg, Phys. Rev. B 39, 274 (1989).

[23] F. C. Zhang and T. M. Rice, Phys. Rev. B 37, 3759 (1988).

[24] Y. Ando, M. Sera, S. Yamagata, S. Kondoh, M. Onoda, and M. Sato, Solid State Commun. 70, 303 (1989). 